\title{
Semiclassical description of resonant tunneling
}

\author{
E.B. Bogomolny and D.C. Rouben \\ Division de Physique Théoriqu日, Institut de Physique Nucléaire \\ 91406 Orsay Cedex, France
}

\begin{abstract}
We derive a semiclassical formula for the tunneling current of electrons trapped in a potential well which can tunnel into and across a wide quantum well. The calculations idealize an experimental situation where a strong magnetic field tilted with respect to an electric field is used. The resulting semiclassical expression is written as the sum over special periodic orbits which hit both walls of the quantum well and are perpendicular to the first wall.
\end{abstract}

PACS numbers: 03.65.Sq, 05.45.+b 
The connection between quantum properties and the underlying classical mechanics has attracted wide attention in the last years (see e.g. [1] and references therein). The central result of this investigation, the Gutzwiller trace formula, relates long-range fluctuations in the density of quantized levels with classical periodic orbits. Each periodic orbit of the classical dynamical system with period $T_{p}$ generates regular maxima in the level density separated by an energy $\Delta E_{p}=\hbar / T_{p}$.

The influence of classical motion on quantum mechanical systems has also been investigated experimentally [2]- [4]. In all cases the observed oscillations were attributed to fluctuations due to special periodic orbits.

In this paper we discuss a particular system to which much experimental work has been devoted in recent years [5] - [8]. The double well potential consisting of GaAs/(AlGa)As resonant tunneling diode containing a wide quantum well $(\mathrm{QW})$ has been used to explore a relationship between classical and quantum electron dynamics. In the presence of a strong uniform magnetic field $(B)$ tilted with respect to the $z$-axis by an angle $\theta$ and a uniform electric field $(\epsilon)$ parallel to the $z$-axis, the system exhibits chaotic motion for certain initial conditions in phase space [5] - [12] and the experiments and numerical simulations reveal oscillations in the tunneling current associated with certain classical periodic orbits [0] - [12]. Even the scarring of wave functions for this problem has been found [8].

The main purpose of this paper is to obtain a quantitative description of the link between the tunneling current and periodic orbits. We shall show that in the strict semiclassical approximation the main contribution will be from special periodic orbits which (i) hit the two walls and (ii) are perpendicular to the first barrier. Unlike the Gutzwiller trace formula the result is not a canonical invariant.

The Hamiltonian (in atomic units) for motion of an electron inside the system, is effectively 2-dimensional and can be written as:

$$
H(y, z)=\frac{\vec{p}^{2}}{2 m}+\frac{B^{2}}{2 m}(y \cos \theta-z \sin \theta)^{2}-\varepsilon z+U(z),
$$

where $U(z)$ is a step-wise potential representing allowed - forbidden layers along $z$ and $m=0.067$ is the band edge mass of an electron in GaAs.

In an over-simplified approximation (cf. [14]) the wave function just after the barrier can be written as a factorized expression corresponding to an approximate separation of variables:

$$
\psi_{1}(y, z)=\frac{C}{\sqrt{k(z)}} \exp \left(\frac{i}{\hbar} \int_{0}^{z} k\left(z^{\prime}\right) d z^{\prime}\right) \psi_{0}(y) .
$$

Here $k(z)$ is $z$-momentum and $\psi_{0}(y)$ is the lowest Landau level eigenfunction for motion in the magnetic field

$$
\psi_{0}(y)=\left(\frac{B \cos \theta}{\pi \hbar}\right)^{1 / 4} \exp \left(-\frac{1}{2 \hbar} B \cos \theta(y-\tilde{y})^{2}\right),
$$

where $\tilde{y}=\langle z>\tan \theta$ is a small shift due to the diagmagnetic term in the perturbation expansion. $|C|^{2}=m \Gamma_{1}$ and $\Gamma_{1}$ is the imaginary part of the energy of the quasi-bound state in the first well computed without the second barrier. 
Using the Stokes theorem one can show that the wave function inside the QW is expressed through its boundary values as follows

$$
\begin{gathered}
\psi(y, z)=\frac{1}{2 m} \int\left(G\left(y, z ; y^{\prime}, 0\right) \partial_{z} \psi_{1}\left(y^{\prime}, 0\right)\right. \\
\left.-\psi_{1}\left(y^{\prime}, 0\right) \partial_{z} G\left(y, z ; y^{\prime}, 0\right)\right) d y^{\prime}
\end{gathered}
$$

where $G\left(y, z ; y^{\prime}, z^{\prime}\right)$ is the Green function for the QW. One can also prove that this result coincides with the first order correction for wave functions in the perturbation theory on the tunneling amplitude similar to one used by Bardeen in [13].

Assuming that the QW is sufficiently wide we shall use the standard semiclassical approximation according to which the Green function is represented by a sum over contributions from classical trajectories $(j)$ connecting final $\vec{x}=(y, z)$ and initial $\overrightarrow{x^{\prime}}=\left(y^{\prime}, z^{\prime}\right)$ points $G\left(y, z ; y^{\prime}, z^{\prime}\right)=\sum_{j} G_{j}\left(\vec{x}, \overrightarrow{x^{\prime}}\right)$, and

$$
G_{j}\left(\vec{x}, \overrightarrow{x^{\prime}}\right)=\frac{m}{i \sqrt{2 \pi i k_{2} k_{1}}}\left|\frac{\partial^{2} S_{j}}{\partial t_{1} \partial t_{2}}\right|^{1 / 2} e^{i \frac{S_{j}}{\hbar}-i \frac{\pi}{2} \mu_{j}}
$$

Here $S_{j}=S_{j}\left(\vec{x}, \overrightarrow{x^{\prime}}\right)$ is the classical action calculated along the classical trajectory $(j)$ connecting initial and final points, $\mu_{j}$ is the Maslov index for this trajectory, $k_{1}$ and $k_{2}$ are the modulus of momentum in the initial and final points respectively, and $t_{1}$ and $t_{2}$ are coordinates perpendicular to the trajectory in these points.

In our case, two modifications have to be made in this standard semiclassical formula. (i) In order to to describe the motion inside a well with finite width walls it is necessary to multiply the expression above by the reflection coefficient. (ii) To take into account various inelastic processes we shall multiply Eq. (5) by a damping factor $\exp \left(-\Gamma_{0} T_{j}\right)$ where $T_{j}$ is the period of the trajectory and $1 / \Gamma_{0}$ plays the role of a mean free time for motion in the QW. In the following we shall ignore the difference between different kinds of mean free paths and in particular the difference between the mean value of the Green function and the mean value of the product of the two Green functions which is important in describing real experiments (see e.g. 四).

The calculation of the Green function when one of its arguments is very close to the boundary of the QW requires special attention. In this case there are 2 different contributions corresponding to 2 trajectories with opposite value of $z$-momentum. The one which hits the boundary has to be multiplied by the reflection coefficient. Therefore $\left.G\left(\vec{x}, \overrightarrow{x^{\prime}}\right)\right|_{z^{\prime}=0}=$ $\left(1+r_{1}\right) G_{j}\left(\vec{x}, \vec{x}_{0}\right)$, and $\left.\partial_{z^{\prime}} G\left(\vec{x}, \overrightarrow{x^{\prime}}\right)\right|_{z^{\prime}=0}=-i p_{z}\left(1-r_{1}\right) G_{j}\left(\vec{x}, \vec{x}_{0}\right)$, where $r_{1}$ is the reflection coefficient from the left wall and $G_{j}\left(\vec{x}, \vec{x}_{0}\right)$ is the contribution of the trajectory starting from the point $\vec{x}_{0}=\left(y^{\prime}, 0\right)$ with $p_{z}>0$ and ending at the point $\vec{x}$.

The explicit expression for the Green function and the value of the wave function in the vicinity of the LH barrier permits one to make a semiclassical computation of the wave function at any point inside the QW. We get

$$
\psi(y, z)=\sum_{j} \int w_{j}\left(y^{\prime}\right) G_{j}\left(y, z ; y^{\prime}, 0\right) d y^{\prime} .
$$

with $w_{j}\left(y^{\prime}\right)=i C v_{j} \sqrt{p_{z}(j)} \psi_{0}\left(y^{\prime}\right) / 2 m$, and $v_{j}=\left(1-r_{1}\right) \sqrt{p_{z}(j) / k_{z}}+\left(1+r_{1}\right) \sqrt{k_{z} / p_{z}(j)}$. Here $p_{z}(j)$ is the initial $z$-momentum of trajectory $j$ and $k_{z}=k(0)$ with $k(z)$ from (2) . Under a 
natural condition $\left|p(j)-k_{z}\right| \ll|p(j)|$ this expression equals 2 and we think that this value is a good zeroth order approximation for this quantity.

Without the simplifying assumption (2)

$$
C \psi_{0}\left(y^{\prime}\right) / \sqrt{k_{z}}=\psi_{1}\left(y^{\prime}, 0\right) \text { and } k_{z}=\partial_{z} \log \psi_{1}\left(y^{\prime}, 0\right)
$$

are just the boundary values of the initial wave function on the LH barrier to be determined either numerically or by more refined methods of multidimensional tunneling.

Knowledge of the wave function (6) permits us to compute the current $\vec{j}_{i}\left(\vec{x}_{f}\right)=\vec{j}_{i}\left(y_{f}, d\right)$ at the second interface of the QW (i.e. at the $\mathrm{RH}$ wall):

$$
\vec{j}_{i}\left(\vec{x}_{f}\right)=\frac{1}{2 m i}\left(\psi^{*}\left(\vec{x}_{f}\right) \vec{\nabla} \psi\left(\vec{x}_{f}\right)-\psi\left(\vec{x}_{f}\right) \vec{\nabla} \psi^{*}\left(\vec{x}_{f}\right)\right) .
$$

When the electron hits the second barrier it has a probability of tunneling through the wall. In the same approximation as above the current after the barrier, $\left(\vec{j}_{f}\right)$, differs from the current before it, $\left(\vec{j}_{i}\right)$, by a transmission coefficient through this barrier $\left(t_{2}\right) \vec{j}_{f}=\left|t_{2}\right|^{2} \vec{j}_{i}$. The total imaginary part, $\Gamma$, of the energy of a quasi-bound state in the emitter well equals the total current after the second barrier

$$
\Gamma=\int_{S} d \overrightarrow{\sigma_{2}} \vec{j}_{i}\left|t_{2}\right|^{2}
$$

Substituting here the expression (6) for the wave function one gets an expression for $\Gamma$ as a triple integral over two initial positions and one final $y$ coordinate:

$$
\Gamma=\sum_{j, k} \int d y d y^{\prime} w_{j k}\left(y, y^{\prime}\right) \int G_{j}\left(y_{f}, y\right) \bar{G}_{k}\left(y_{f}, y^{\prime}\right) d y_{f}\left|t_{2}\right|^{2}
$$

where $w_{j k}\left(y, y^{\prime}\right)=\left(p_{z}^{(f)}(j)+p_{z}^{(f)}(k)\right) w_{j}(y) \bar{w}_{k}\left(y^{\prime}\right) / 2 m$ and $G_{j}\left(y_{f}, y\right)$ is the contribution (5) of a trajectory $(j)$ which starts at point $(y, 0)$ and ends at point $\left(y_{f}, d\right)$.

In the semiclassical approximation $G_{j}$ is proportional to $\exp \left(i S_{j} / \hbar\right)$ and in the formal limit $\hbar \rightarrow 0$ it is natural to perform the integration over all variables by the saddle point method. Assuming that the boundary function $\psi_{0}(y)$ and other quantities are smooth in the scale of noticeable changes of the Green function, one concludes that in such an approximation the dominant contribution to the above integrals will be given by trajectories in the vicinity of saddle-points trajectories for which the following three conditions are fulfilled:

$$
\begin{gathered}
\partial S_{j}\left(y_{f}, y\right) / \partial y_{f}-\partial S_{k}\left(y_{f}, y^{\prime}\right) / \partial y_{f}=0 \\
\partial S_{j}\left(y_{f}, y\right) / \partial y=0, \partial S_{k}\left(y_{f}, y^{\prime}\right) / \partial y^{\prime}=0 .
\end{gathered}
$$

The first equation means that saddle point trajectories (labeled here by $j$ and $k$ ) should have the same $y$ component of momentum: $p_{y}^{(f)}(j)=p_{y}^{(f)}(k)$. The equality of the $p_{z}$ momenta for these trajectories then follows from energy conservation. But two classical trajectories passing through the same point $\left(y_{f}\right)$ and having the same momenta at this point can be either (i) exactly the same trajectory or (ii) two different paths on the same classical trajectory.

The second pair of saddle point equations (11) signifies that the saddle point trajectories should have zero $y$ component of the momentum in both points at the LH wall (i.e. they 
have to be perpendicular to the plane $z=0$ ). The combination of these conditions leads to the important conclusion that in the strict semiclassical limit the tunneling current or probability of decay is divided into two distinct contributions: $\Gamma=\Gamma_{(\text {Weyl })}+\Gamma_{(o s c)}$. The first term corresponds to the interference of an arbitrary trajectory perpendicular to the plane $z=0$ with itself. It has no quick dependence on external fields and in real experiments it is effectively washed out by taking the second derivative of the current.

The second term, which we denote by $\Gamma_{(o s c)}$, includes the contributions from different paths on the same self-retracing periodic trajectory which connect the LH and RH walls and is perpendicular to the plane $z=0$. The classical trajectories which give the dominant contribution to the resonant tunneling are built from a segment of a periodic orbit which hits both walls plus an arbitrary number of loops $(n)$ around this orbit.

Expanding the actions up to the quadratic terms, expressing the derivatives of actions through the monodromy matrix, performing the sum over all possible repetition numbers one gets

$$
\begin{aligned}
& \Gamma_{(\text {osc })}=\sum_{p} \Gamma_{p} \frac{\left|t_{2}^{(t o t)}(p)\right|^{2}}{1-\left|R_{p}\right|^{2}} \times \\
& \sum_{r=1}^{\infty} \frac{R_{p}^{r}}{D(r)^{1 / 2}} \exp \left(i S(r)-i \frac{\pi}{2} \mu_{p}(r)+i \frac{\pi}{4}\right)+c . c .
\end{aligned}
$$

where $D(r)=m_{21}(r)$ and $S(r)=r S_{p}$. The sum here is taken over all primitive periodic orbits with $p_{y}(i)=0$ and over up to 2 points of reflection of this trajectory with the first wall: $y_{0}$ and $y_{0}^{\prime} . S_{p}$ is the classical action around the periodic orbit labeled by $p$ [15], $m_{i j}(r)$ is the $(i, j)$ monodromy matrix element computed along the first barrier for $r$ repetitions of the orbit considered, $R_{p}=r_{(t o t)}(p) \exp \left(-\Gamma_{0} T_{p}\right)$ where $r_{(t o t)}(p)$ is the total reflection coefficient from the walls for one loop around the given orbit, $\left|t_{2}^{(t o t)}(p)\right|^{2}=\sum_{l}\left|t_{2}(p, l)\right|^{2} \exp \left(-2 \Gamma_{0} T_{0}(l)\right)$ is the total tunneling probability through the second barrier weighted by damping factors, and $\Gamma_{p}=(\pi / 8)^{1 / 2} v_{p} \bar{v}_{p}^{\prime} \Gamma_{1} \psi_{0}\left(y_{0}\right) \bar{\psi}_{0}\left(y_{0}^{\prime}\right)$.

The formula, Eq. (12), is the main result of the paper. It expresses the semiclassical limit of the tunneling current (which is proportional to $\Gamma$ ) as a sum over special periodic trajectories which (i) connect the two walls of the QW and (ii) are perpendicular to the first barrier.

The important ingredient in deriving the above formula was the assumption related to the applicability of the semiclassical approximation. Whereas for the integration over the final coordinate it can easily be argued, the requirement that the initial wave function changes slowly in the semiclassical limit is more difficult to justify. The main problem is that the Landau-type wave function (3) has the term $B \cos \theta y^{2} / 2 \hbar$ in the exponent and both terms, one coming from the initial wave function and one from the semiclassical Green function, have the same dependance on $\hbar$. In this case one can proceed as follows. It is easy to check (see (14)) that the requirement of the smoothness of the initial wave function is equivalent to the condition

$$
\beta \ll \partial^{2} S\left(y, y^{\prime}\right) / \partial y^{2}=m_{11} / m_{12}
$$

where $\beta=B \cos \theta$ and the same condition for the derivative over $y^{\prime}$. 
But the value of the magnetic field in dimensionless units is very small. On the other hand for generic chaotic systems all elements of the monodromy matrix should be of the same order and there is no general reason why the above ratio should be small. Therefore we expect that for hyperbolic periodic orbits with large Lyapunov exponent the condition (13) will be satisfied. We have checked numerically that the ratio $m_{11} /\left(m_{12} \beta\right)$ grows quickly with increasing $m_{11}$ though for stable and almost stable orbits it can be of the order of 1 .

To treat the latter case a slightly different approximation can be used. Let us expand the action $S\left(y, y^{\prime}\right)$ up to the second order and perform the integration over $y$ and $y^{\prime}$ in (9) taking into account explicitly the dependance of the initial wave function (3) on these variables. After simple algebra we get the same expression as in (12) but with the pre-factor $D(r)$ and the action $S(r)=r S_{p}+\Delta S(r)$ substituted by

$$
\begin{aligned}
D(r) & =m_{21}(r)+i \beta\left(m_{11}(r)+m_{22}(r)\right)-\beta^{2} m_{12}(r), \\
\Delta S(r) & =\frac{\beta^{2}}{2 D(r)}\left(y_{0}^{2}\left(m_{22}(r)+i \beta m_{12}(r)\right)\right. \\
& \left.+y_{0}^{\prime 2}\left(m_{11}(r)+i \beta m_{12}(r)\right)+2 y_{0} y_{0}^{\prime}\right) .
\end{aligned}
$$

These formulae are valid provided the third and higher terms inn the expansion of the action are small in comparison with quadratic terms.

When $\beta \rightarrow 0$ this result leads to Eq. (12). But for stable and almost stable regions where condition (13) is not well satisfied Eqs. (12) with (14) continue to be a good approximation for $\Gamma$ in Eq. (9). In such cases one can (exactly or approximately) split the Hamiltonian into the sum of two Hamiltonians for longitudinal and transverse motion and the energy of the former is much bigger than the latter. Under these conditions (i) the period of motion is defined mainly by the longitudinal motion and (ii) the transverse motion is close to the motion in a quadratic potential similar to (II) with $\theta=0$ for a fixed time. But it is known [16] that the semiclassical time-dependent Green function for the motion in a quadratic potential coincides with the exact Green function and both are proportional to the exponential of a function quadratic in its coordinates. This means that for stable and almost stable regions there exist good reasons why higher than quadratic terms in the expansion of the action on transverse coordinates should be small and, therefore, Eqs. (12) and (14) are good approximations to the triple integral in (9). Of course, near bifurcations when by, definition, the quadratic form in the exponent is degenerate these equations require modification.

There is an interesting limit of the above formulae. It corresponds to very clean devices where $\Gamma_{0}$ is small and the probability of the tunneling through the second wall is much bigger than through the fist one. In this case $R$ equals the reflection coefficient form the second wall and using the relation $|r|^{2}=1-|t|^{2}$ one concludes that the factor $\left|t_{2}^{(t o t)}\right|^{2} /\left(1-|R|^{2}\right)$ in Eq. (12) tends to 1. One can show that all formulae in such a limit coincide with the semiclassical limit of a simplified model of resonant tunneling discussed in [11] and [12 which is very convenient for numerical simulation. This model is based on the Bardeen transfer matrix [13 according to which the probability of tunneling (or the imaginary part of the energy level) is given by

$$
\Gamma=2 \pi \sum_{n}\left|W_{n}\right|^{2} \delta\left(E-E_{n}\right)
$$


where $E_{n}$ denotes the exact energy levels in the quantum well and the coefficients $W_{n}$ are the matrix elements of the current between the wave function in the first well $\left(\psi_{1}\right)$ and the exact wave function $\left(\psi_{n}(y)\right)$ in the QW

$$
W_{n}=-\frac{i}{2 m} \int\left(\frac{\partial \bar{\psi}_{1}(\vec{q})}{\partial z} \psi_{n}(\vec{q})-\bar{\psi}_{1}(\vec{q}) \frac{\partial \psi_{n}(\vec{q})}{\partial z}\right) d y
$$

with the $z$-component of point $\vec{q}=(y, z)$ somewhere inside the barrier.

Rewriting Eqs. (15) and (16) through the Green function and its $z$-derivatives and using the same arguments as above it is possible to demonstrate that the semiclassical approximation to the resulting expression are given by Eqs. (12) with $\left|t_{2}^{(t o t)}\right|^{2} /\left(1-|R|^{2}\right)=1$.

The same formulae can be used to derive an approximation similar to the Miller modification of the Gutzwiller trace formula [17 for a stable orbit. In such a case the monodromy matrix elements for $r$ repetitions around a primitive periodic orbit are functions of $\exp (i \omega r)$ where $\exp ( \pm i \omega)$ are eigenvalues of monodromy matrix. By representing Eqs. (12) and (14) as a power series $\sum_{m} \exp (-i \omega r m) C_{m}$ and performing the summation over $r$ one gets an expression of $\Gamma$ as a sum over $m$ :

$$
\begin{aligned}
\Gamma & =\frac{\pi(\beta \rho)^{1 / 2}}{1+\beta \rho} \frac{\Gamma_{1}}{T} \exp \left(-\frac{\beta}{2(1+\beta \rho)}\left(y_{0}^{2}+y_{0}^{\prime 2}\right)\right)\left|v_{p}\right|^{2} \\
& \times \sum_{m} \frac{1}{2^{m} m !}\left(\frac{\beta \rho-1}{\beta \rho+1}\right)^{m} H_{m}\left(\lambda y_{0}\right) H_{m}\left(\lambda y_{0}^{\prime}\right) \\
& \times \sum_{N} \delta\left(E-E_{N, m}\right),
\end{aligned}
$$

where $\rho=m_{12} / \sin \omega, \lambda=\beta \sqrt{\rho /\left(\beta^{2} \rho^{2}-1\right)}$ and $E_{N, m}$ are approximate eigenvalues defined as in [17]: $S_{p}\left(E_{N, m}-\epsilon_{\perp}(m)\right)=2 \pi \hbar\left(N+\mu_{p} / 4\right)$ and $\epsilon_{\perp}=\hbar \omega(m+1 / 2)$.

The index $m$ has the meaning of the number of states in an effective transversal Hamiltonian

$$
H(p, y)=\omega\left(\rho p^{2}+y^{2} / \rho\right) / 2 .
$$

and the resulting expression corresponds to Eqs. (15) and (16) in which one approximates $\psi_{n}(y, z)$ by a product $\psi(z) \psi_{m}(y)$ where $\psi_{m}(y)$ are eigenfunctions of this Hamiltonian. By truncating this sum at a finite value of $m$ one obtains the Miller-type approximation which generalizes the tori quantization discussed in [12].

In conclusion we have developed a simple semiclassical theory of resonant tunneling. In the strict semiclassical limit the tunneling current is due to only special periodic orbits in the QW which are perpendicular to the LH wall and hit the $\mathrm{RH}$ wall of the QW, and their contributions are proportional to $\left(m_{21}\right)^{-1 / 2}$. For almost stable orbits one has to use Eq. (14) but the period of oscillations will deviate slightly from that of periodic orbits. For big stable regions the Miller-type formula (17) is appropriate.

It is a pleasure to acknowledge D. Delande and T. Monteiro for many useful discussions and for presenting us papers 11] and 12 prior to their publication. The authors would also like to thank S.C. Creagh, A. Mouchet, D. Saraga, M. Sieber, N.D. Whelan, and D. Ullmo for stimulating discussions. D.C.R. was supported by the Natural Sciences and Engineering Research Council of Canada Fellowship (PGS-B award, No. 148961). 


\section{REFERENCES}

* Unité de Recherche des Universités Paris 11 et Paris 6, Associée au CNRS

[1] Les Houches Summer School, 1989, Chaos and Quantum Physics, edited by M.J. Giannoni, A. Voros, and J. Zinn-Justin (Elsevier, New York, 1991)

[2] H. Friedrich and D. Wintgen, Phys. Rep. 183, 37 (1989)

[3] H.U. Baranger et al, Phys. Rev. Lett. 70, 3876 (1993)

[4] K. Richter et al, Phys. Rep. 276, 1 (1996)

[5] T.M. Fromhold et al, Phys. Rev. Lett. 72, 2608 (1994)

[6] T.M. Fromhold et al, Phys. Rev. Lett. 75, 1142 (1995)

[7] G. Müller et al, Phys. Rev. Lett. 75, 2875 (1995)

[8] P.B. Wilkinson et al, Nature 380, 608 (1996)

[9] D.L. Shepelyansky et al, Phys. Rev. Lett. 74, 2098 (1974)

[10] T.S. Monteiro et al, Phys. Rev. E 53, 3369 (1996)

[11] T.S. Monteiro et al, Phys. Rev. B 56, 3913 (1997)

[12] T.S. Monteiro and D. Saraga, (1997) to be published

[13] J. Bardeen, Phys. Rev. Lett. 6, 57 (1966)

[14] M.L. Leadbeater et al, Sem. Sci. Tec. 6, 1021 (1991)

[15] When $y_{0} \neq y_{0}^{\prime} S_{p}$ corresponds to a half of periodic orbit.

[16] R.P. Feymann and A.R. Hibbs, Quantum mechanics and Path Integrals (McGraw-Hill Publishing Company, 1965)

[17] W.Miller, J. Chem. Phys., 63, 996 (1975) 